\title{
Birth cohort-specific trends of sun-related behaviors among individuals from an international consortium of melanoma- prone families
}

John Charles A. Lacson ${ }^{1 \dagger}$, Shawn A. Zamani ${ }^{1,2+}$, Luis Alberto Ribeiro Froes $\mathrm{Jr}^{3} \mathbb{B}^{\mathrm{B}}$, Nandita Mitra ${ }^{4}$, Lu Qian ${ }^{5}$, Scarlet H. Doyle ${ }^{1}$, Esther Azizi ${ }^{6,7}$, Claudia Balestrini ${ }^{8}$, D. Timothy Bishop ${ }^{9}$, William Bruno ${ }^{10}$, Blanca Carlos-Ortega ${ }^{11}$, Francisco Cuellar ${ }^{12,13}$, Anne E. Cust ${ }^{14,15}$, David E. Elder ${ }^{16}$, Anne-Marie Gerdes ${ }^{17}$, Paola Ghiorzo ${ }^{10,18}$, Thais C. Grazziotin ${ }^{19}$, Nelleke A. Gruis ${ }^{20}$, Johan Hansson ${ }^{21}$, Marko Hočevar ${ }^{22}$, Veronica Höiom ${ }^{21}$, Elizabeth A. Holland ${ }^{23}$, Christian Ingvar ${ }^{24}$, Gilles Landman ${ }^{25,26}$, Alejandra Larre-Borges ${ }^{27}$, Graham J. Mann ${ }^{15,23,28}$, Montserrat Molgo ${ }^{29}$, Luciana Facure Moredo ${ }^{30}$, Håkan Olsson ${ }^{24}$, Jacoba J. Out-Luiting ${ }^{20}$, Barbara Perić22, Dace Pjanova ${ }^{31}$, Susana Puig ${ }^{12,32,33}$, Julio Salas-Alanis ${ }^{11,34}$, Helen Schmid ${ }^{23}$, Karin A. W. Wadt ${ }^{17}$, Julia A. Newton-Bishop ${ }^{9}$, Peter A. Kanetsky ${ }^{1 *}$ (I) and on behalf of the GenoMEL Study Group

\begin{abstract}
Background: Individuals from melanoma-prone families have similar or reduced sun-protective behaviors compared to the general population. Studies on trends in sun-related behaviors have been temporally and geographically limited.
\end{abstract}

Methods: Individuals from an international consortium of melanoma-prone families (GenoMEL) were retrospectively asked about sunscreen use, sun exposure (time spent outside), sunburns, and sunbed use at several timepoints over their lifetime. Generalized linear mixed models were used to examine the association between these outcomes and birth cohort defined by decade spans, after adjusting for covariates.

(Continued on next page)

\footnotetext{
* Correspondence: Peter.Kanetsky@moffitt.org

John Charles A Lacson and Shawn A Zamani are joint first authors.

${ }^{\dagger}$ John Charles A. Lacson and Shawn A. Zamani contributed equally to this

work.

'Department of Cancer Epidemiology, Division of Population Science, H. Lee

Moffitt Cancer Center \& Research Institute, 12902 Magnolia Dr., MRC 213,

Tampa, FL 33612, USA

Full list of author information is available at the end of the article
}

(c) The Author(s). 2021 Open Access This article is licensed under a Creative Commons Attribution 4.0 International License, which permits use, sharing, adaptation, distribution and reproduction in any medium or format, as long as you give appropriate credit to the original author(s) and the source, provide a link to the Creative Commons licence, and indicate if changes were made. The images or other third party material in this article are included in the article's Creative Commons licence, unless indicated otherwise in a credit line to the material. If material is not included in the article's Creative Commons licence and your intended use is not permitted by statutory regulation or exceeds the permitted use, you will need to obtain permission directly from the copyright holder. To view a copy of this licence, visit http://creativecommons.org/licenses/by/4.0/ The Creative Commons Public Domain Dedication waiver (http://creativecommons.org/publicdomain/zero/1.0/) applies to the data made available in this article, unless otherwise stated in a credit line to the data. 
(Continued from previous page)

Results: A total of 2407 participants from 547 families across 17 centers were analyzed. Sunscreen use increased across subsequent birth cohorts, and although the likelihood of sunburns increased until the 1950s birth cohort, it decreased thereafter. Average sun exposure did not change across the birth cohorts, and the likelihood of sunbed use increased in more recent birth cohorts. We generally did not find any differences in sun-related behavior when comparing melanoma cases to non-cases. Melanoma cases had increased sunscreen use, decreased sun exposure, and decreased odds of sunburn and sunbed use after melanoma diagnosis compared to before diagnosis.

Conclusions: Although sunscreen use has increased and the likelihood of sunburns has decreased in more recent birth cohorts, individuals in melanoma-prone families have not reduced their overall sun exposure and had an increased likelihood of sunbed use in more recent birth cohorts. These observations demonstrate partial improvements in melanoma prevention and suggest that additional intervention strategies may be needed to achieve optimal sun-protective behavior in melanoma-prone families.

Keywords: Trends, Sun-related behaviors, Sunscreen use, Sun exposure, Sunburn, Sunbed, Melanoma, High-risk families, Skin Cancer

\section{Background}

The incidence of cutaneous malignant melanoma has been increasing over the past 50 years in populations that are predominantly fair-skinned [1]. Although rates have stabilized recently in several parts of the world including Australasia and North America, they continue to rise in most European and South American countries [1-3]. Exposure to ultraviolet radiation, especially intermittent sun exposure resulting in sunburn, is the main environmental factor associated with increased melanoma risk [4].

In the general population, sun-protective behavior has improved, evidenced by increased sunscreen use and increased sun protection factor (SPF) [5-8]. However, among individuals with a family history of melanoma, who have double to quadruple the risk of developing melanoma compared to the general population [9], sun-seeking and tanning behaviors are still prevalent [10]. In addition, sunprotective behaviors are suboptimal among children and adolescents who have a family history of melanoma [1013] and among adolescents in the general population [14, 15]. These deficits are notable because ultraviolet radiation exposure during childhood and adolescence is a strong determinant of melanoma risk [16, 17].

Previous studies on sun-protective behaviors in the general population were limited to a specific geographic region, examined trends only within a single or a few decades, or did not examine behaviors throughout the lifetime of participants $[5,6,14,18-$ 25]. Studies of sun-related behaviors among individuals with a family history of melanoma mainly focused on recent exposure and rarely describe trends over time [10, 12, 26-28].

We report results from a large international study of melanoma-prone families, in which participants were queried about sun-related behaviors at several points over their lifetime, including childhood and adolescence.
We characterize trends in sun-related behavior over most of the twentieth century by birth cohort and compare between melanoma cases and non-cases. Among cases, we also compare these behaviors before and after melanoma diagnosis.

\section{Methods \\ Study population}

The Melanoma Genetics Consortium, GenoMEL, recruited individuals from melanoma-prone families at 27 centers across the globe. Our study sample included participants from 17 GenoMEL centers in Europe, North and South America, Australia, and the Middle East. Melanoma-prone families were defined by the presence of three or more melanoma cases among blood relatives, or two or more cases in first-degree relatives. Personal history of melanoma was self-reported or verified from pathology report, cancer registry, clinical notation, or death certificate. Participants were mailed a questionnaire that solicited information on demographics, personal history of melanoma, phenotypic variables, and a personal residence calendar that captured yearly information on location of residence, school and/or work, and the average number of days per week spent at school and/or work (Additional file 1). A subsequent questionnaire administered by GenoMEL research staff recorded details on personal sun exposure and sunprotective behaviors at multiple time points across the life course, using the residence and school/work calendar as memory prompts (Additional file 2). This instrument was adapted from one used in several epidemiological studies of melanoma and has been previously validated $[29,30]$.

\section{Demographics and phenotypic covariates}

Information on sex and date of birth were obtained. Individuals were grouped into decade birth cohorts 
starting with the 1910s and ending with the 1980s. We combined the participants born in 1910s $(n=15)$ with those born in 1920s $(n=136)$. Participants born in the 1990s $(n=29)$ or 2000 s $(n=1)$ were excluded due to limited lifecourse information. Phenotypic information was collected on hair color (red, blonde, light brown, dark brown, black), eye color (blue, brown/black, other), and skin complexion (very fair, fair, olive, brown, black, other). Participants reported burn severity after an hour of mid-day summer sun exposure without protection (severe sunburn with skin blistering, painful sunburn with skin peeling, mildly sunburned with some tanning, or no sunburn with brown tan), and tanning after repeated sun exposure without protection (deep brown tan, moderate tan, mild tan, or no tan/only freckling). Additionally, participants reported face freckling in childhood and as an adult (none, very few, few, some, many, very many), and the number of moles on the body (none, few, many, very many).

\section{Sunscreen use, sun exposure, sunburn, and sunbed use}

Information on sunscreen use, sun exposure, and sunburns was obtained at anchor years of 10, 15, 20, 30, 40 years old, and the year before the completion date of the survey questionnaire. For each anchor year, participants were asked about the frequency of sunscreen use while exposed to sunlight on a five-point scale $(1$ - never or hardly ever, 2 - less than half the time, 3 - about half the time, 4 - more than half the time, 5 - always or almost always). For participants who reported sunscreen use, use of sunscreen with a SPF of 8 or higher (yes, no) was assessed. A SPF-weighted sunscreen variable was formulated by assigning individuals who reported using SPF less than 8 to having never used sunscreen during that timepoint.

At each anchor year, participants were asked to report personal outdoor sun exposure and the number of painful sunburns that lasted for two or more days. We obtained detailed information on number of hours spent outside between $9 \mathrm{AM}$ and $5 \mathrm{PM}$ during weekdays, weekends, and holidays, separately for warmer and colder months. Holidays were defined as days spent away from school or work.

Daily average sun exposure (time spent outside in hours) was calculated separately for weekdays, weekends, and holidays, combining responses from warmer and colder months within each anchor year. To calculate overall sun exposure, a time-weighted integration of hours spent outdoors was calculated based on 5 days for weekdays and 2 days for weekend days during work and school weeks, and 7 days for holiday weeks over the 26 weeks for warmer and colder months, respectively. Hours of warmer and colder months were then averaged to generate outdoor hours for the entire year. To calculate lifetime average sun exposure, data from each anchor year were assigned to a specific period of life: anchor year 10 was assigned to ages 5 to 12 , anchor year 15 to ages 13 to 17 , anchor year 20 to ages 18 to 24 , anchor year 30 to ages 25 to 34, anchor year 40 to ages 35 to 44 , and the last anchor year to ages 45 and above. Sun exposure measurements were then averaged over the number of years recorded for each individual. The following imputation scheme was used for missing values: if either the first or last anchor year was missing, the closest non-missing value was carried backward or forward. If a missing anchor year was between two nonmissing values, the average of the two adjacent nonmissing values was used. Imputation was done separately for weekdays, weekends, and holidays, within warmer and colder months.

Participants reported sunlamp or sunbed use on more than one occasion (ever, never). This question was asked only once covering the entire life course and not at each anchor year. Participants who responded an ever-use of sunlamps or sunbeds were asked about their age at firstand last-use.

\section{Statistical analyses \\ Factor analysis of phenotypic variables}

Due to the high covariance of phenotypic variables, factor analysis was performed. Following components extraction using principal axis method with Varimax (orthogonal) rotation, three meaningful factors emerged and were retained for rotation. Each of these components had an eigenvalue $>1.00$ and have a cumulative percent variance $>70 \%$. Each variable was loaded on a component if the rotated factor pattern loading was greater than 0.40 for that factor and less than 0.40 for the other two factors. Using this scheme, burnability, tanability, skin complexion loaded on the first factor; hair color, eye color, extent of body moles loaded on the second; and childhood and adulthood face freckles loaded on the third. Factor-based scores were calculated per participant. Factors were modeled in a confirmatory factor analysis and robust maximum likelihood estimation yielded a good fit with the model (RMSEA $=0.05$; $\mathrm{CFI}=0.97$; SRMR $=0.03$ ). We tested for heterogeneity of trend by pre- vs. post-diagnosis by introducing an interaction term between diagnostic period and birth cohort. All statistical analyses were conducted using SAS v. 9.4 (Statistical Analysis System, RRID:SCR_008567).

\section{Life course and age group analyses}

All statistical analyses were conducted using SAS v. 9.4 (Statistical Analysis System, RRID:SCR_008567). Predicted population marginal means were estimated using SAS GLIMMIX procedure; separate models were used for sunscreen use (including SPF-weighted use), hours of 
sun exposure, ever having sunburns, or ever use of sunbeds as the outcome and birth cohort as the predictor (categorical) variable. We conducted trend analyses by coding birth cohort as an ordinal variable in the model. All models included random effects to account for clustering within family, and we used empirical (sandwich) variance estimators. In the models, sunscreen data were assumed to follow a gamma distribution, and sun exposure data were assumed to follow a normal distribution. Due to sparse data, we modeled sunburn data as a binomial outcome. Analyses of sunscreen use commenced with the 1950 birth cohort, which corresponds to the decade sunscreen became commercially available [31].

All models were adjusted for sex, center (grouped by latitude: high northern, mid-high northern, mid northern, southern), and the three factor variables for phenotype. We conducted analyses for the entire life course and within three life periods: 10 years old or younger, 11 to 20 years, and after 20 years old. Life course models and models for after 20 years old also included a covariate capturing number of years unaffected with melanoma, which was equal to the age at diagnosis for cases, or to the age at interview for non-cases.

\section{Analyses stratified by melanoma status, and before and after diagnosis}

We used the same models as above to obtain predicted population marginal means and perform tests of trends after stratifying by melanoma status. We tested for the difference of each outcome variable by melanoma status across the birth cohorts by adding melanoma status as a covariate to the full model. Tests of heterogeneity of trend were conducted by adding an interaction term between birth cohort and melanoma status.

For melanoma cases, all outcome variables were further defined by whether they occurred before or after diagnosis and were compared using the SAS GLIMMIX procedure. Random effects were included in the model to account for clustering of individuals within families. Because of implicit individual-matching across the preand post-diagnostic periods, other covariates were not included in these models. We tested for heterogeneity of trend by pre- vs. post-diagnosis by introducing an interaction term between diagnostic period and birth cohort.

\section{Results}

A total of 2407 participants from 547 families across 17 centers were recruited (Table 1). The mean age of participants was 52.9 years old $(\mathrm{SD}=16)$, and $59 \%$ were female (Table 2 ). The majority $(60 \%)$ of participants were born in the 1940s, 1950s, or 1960s, with approximately $20 \%$ in each decade. Almost half (47.8\%) of participants reported at least one primary cutaneous melanoma.

\section{Sunscreen use}

We noted a clear secular trend in average reported frequency of sunscreen use with nearly identical increases over time across all birth cohorts (Fig. 1a). Sunscreen use increased as each birth cohort aged, and more recent cohorts had increased sunscreen use at younger ages

Table 1 Number of families, individual, and melanoma affected or unaffected individuals per GenoMEL center

\begin{tabular}{|c|c|c|c|c|c|}
\hline Latitude & Center & Families & Individuals & Unaffected & Affected \\
\hline \multirow[t]{3}{*}{ High northern } & Copenhagen, DK & 26 & 52 & 0 & 52 \\
\hline & Lund, SE & 6 & 41 & 25 & 16 \\
\hline & Stockholm, SE & 22 & 125 & 89 & 36 \\
\hline \multirow[t]{4}{*}{ Mid-high northern } & Leeds, UK & 93 & 303 & 139 & 164 \\
\hline & Leiden, NL & 47 & 431 & 309 & 122 \\
\hline & Ljubljana, SI & 6 & 16 & 2 & 14 \\
\hline & Riga, LV & 3 & 8 & 5 & 3 \\
\hline \multirow[t]{4}{*}{ Mid northern } & Barcelona, ES & 35 & 147 & 91 & 56 \\
\hline & Genoa, IT & 25 & 79 & 25 & 54 \\
\hline & Philadelphia, US & 31 & 95 & 31 & 64 \\
\hline & Tel Aviv, IL & 31 & 127 & 66 & 61 \\
\hline \multirow[t]{6}{*}{ Southern } & Mexico City, MX & 2 & 9 & 5 & 4 \\
\hline & Montevideo, UY & 9 & 55 & 42 & 13 \\
\hline & Porto Alegre, BR & 17 & 40 & 16 & 24 \\
\hline & Santiago, CL & 4 & 10 & 2 & 8 \\
\hline & São Paulo, BR & 15 & 65 & 36 & 29 \\
\hline & Sydney, AU & 175 & 804 & 374 & 430 \\
\hline Total & 17 & 547 & 2407 & 1257 & 1150 \\
\hline
\end{tabular}


Table 2 Characteristics of GenoMEL participants $(N=2407)$

\begin{tabular}{|c|c|c|}
\hline Variable & Individuals, $n$ (\%) & \\
\hline \multicolumn{3}{|l|}{ Age at Interview } \\
\hline Mean (SD) & 52.9 & (16) \\
\hline$<20$ & 16 & $(0.7)$ \\
\hline $20-30$ & 182 & $(7.6)$ \\
\hline $30-40$ & 340 & (14.1) \\
\hline $40-50$ & 478 & (19.9) \\
\hline $50-60$ & 514 & (21.4) \\
\hline $60-70$ & 459 & (19.1) \\
\hline $70-80$ & 319 & (13.3) \\
\hline $80+$ & 99 & $(4.2)$ \\
\hline \multicolumn{3}{|l|}{ Birth decade } \\
\hline 1910-1929 & 147 & $(6.1)$ \\
\hline 1930-1939 & 351 & (14.6) \\
\hline 1940-1949 & 493 & (20.5) \\
\hline 1950-1959 & 501 & $(20.8)$ \\
\hline 1960-1969 & 482 & (20.0) \\
\hline 1970-1979 & 292 & (12.1) \\
\hline 1980-1989 & 141 & (5.9) \\
\hline \multicolumn{3}{|l|}{ Sex } \\
\hline Female & 1421 & $(59.0)$ \\
\hline Male & 986 & $(41.0)$ \\
\hline \multicolumn{3}{|l|}{ Hair color } \\
\hline Red & 279 & (11.6) \\
\hline Blonde or fair & 848 & $(35.2)$ \\
\hline Brown & 1164 & (48.4) \\
\hline Black & 104 & $(4.3)$ \\
\hline Missing & 12 & $(0.5)$ \\
\hline \multicolumn{3}{|l|}{ Eye color } \\
\hline Blue & 874 & (36.3) \\
\hline Gray, hazel or green & 1062 & $(44.1)$ \\
\hline Brown or black & 464 & (19.3) \\
\hline Missing & 7 & $(0.3)$ \\
\hline \multicolumn{3}{|l|}{ Skin complexion } \\
\hline Very fair & 490 & $(20.4)$ \\
\hline Fair & 1699 & (70.6) \\
\hline Darker $^{a}$ & 205 & $(8.5)$ \\
\hline Missing & 13 & $(0.5)$ \\
\hline \multicolumn{3}{|l|}{ Burnability } \\
\hline Severe burn, blister & 194 & (8.1) \\
\hline Painful burn, peel & 1032 & $(42.9)$ \\
\hline Mild burn, some tan & 959 & (39.8) \\
\hline No burn, brown tan & 165 & $(6.9)$ \\
\hline Missing & 57 & (2.4) \\
\hline
\end{tabular}


Table 2 Characteristics of GenoMEL participants $(N=2407)$ (Continued)

\begin{tabular}{|c|c|c|}
\hline Variable & Individuals, $n$ (\%) & \\
\hline \multicolumn{3}{|l|}{ Tanability } \\
\hline No tan or get freckled & 266 & $(11.1)$ \\
\hline Moderate tan & 1045 & $(43.4)$ \\
\hline Mild or occasional tan & 761 & (31.6) \\
\hline Deep tan & 272 & $(11.3)$ \\
\hline Missing & 63 & (2.6) \\
\hline \multicolumn{3}{|l|}{ Childhood face freckles } \\
\hline Very many & 40 & $(1.7)$ \\
\hline Many & 176 & (7.3) \\
\hline Some & 304 & (12.6) \\
\hline Few & 464 & (19.3) \\
\hline Very few & 665 & $(27.6)$ \\
\hline None & 723 & (30.0) \\
\hline Missing & 35 & $(1.5)$ \\
\hline \multicolumn{3}{|l|}{ Adulthood face freckles } \\
\hline Very many & 23 & $(1.0)$ \\
\hline Many & 77 & $(3.2)$ \\
\hline Some & 211 & $(8.8)$ \\
\hline Few & 343 & $(14.3)$ \\
\hline Very few & 798 & $(33.2)$ \\
\hline None & 916 & $(38.1)$ \\
\hline Missing & 39 & $(1.6)$ \\
\hline \multicolumn{3}{|l|}{ Extent of body moles } \\
\hline Many & 530 & $(22.0)$ \\
\hline Some & 916 & $(38.1)$ \\
\hline Few & 760 & (31.6) \\
\hline None & 167 & (6.9) \\
\hline Missing & 34 & $(1.4)$ \\
\hline \multicolumn{3}{|l|}{ Melanoma history } \\
\hline Affected & 1150 & $(47.8)$ \\
\hline Unaffected & 1257 & $(52.2)$ \\
\hline \multicolumn{3}{|l|}{ Center latitude } \\
\hline High northern & 218 & $(9.1)$ \\
\hline Mid-high northern & 758 & (31.5) \\
\hline Mid northern & 448 & (18.6) \\
\hline Southern & 983 & $(40.8)$ \\
\hline
\end{tabular}

${ }^{a}$ Darker skin includes olive, brown, black and other

compared to earlier birth cohorts. Results were similar after adjusting for SPF (data not shown).

Adjusted average lifetime sunscreen use increased by birth cohort (per birth cohort $\beta=0.08, P=0.004$ ), and this trend strengthened after weighting by $\operatorname{SPF}(\beta=0.11$, $P=0.0003$, Table 3$)$. This trend of increasing sunscreen with subsequent birth cohort was evident within each life period, within strata defined by melanoma status, and before and after melanoma diagnosis. We found no difference or heterogeneity in trends when comparing cases and non-cases. Cases had higher average sunscreen use (adjusted mean difference $(\mathrm{AMD})=1.36, P<0.0001$ ) after diagnosis than before diagnosis. All results were similar after weighting by SPF. 
(a)

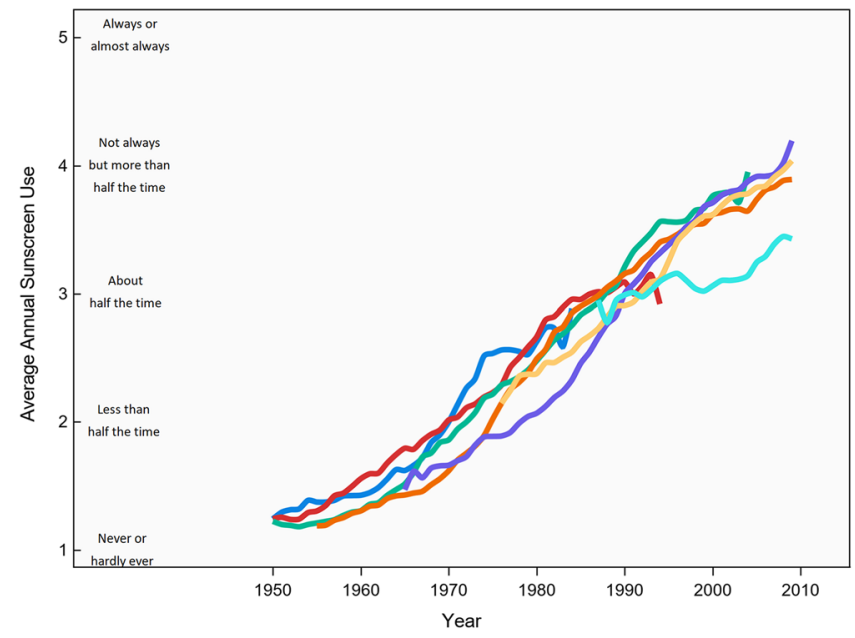

(b)

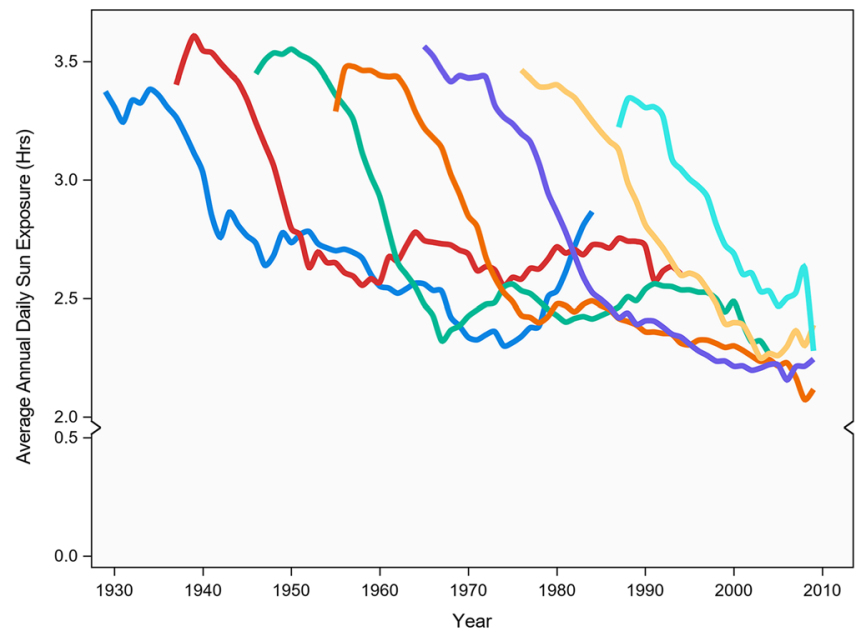

(c)

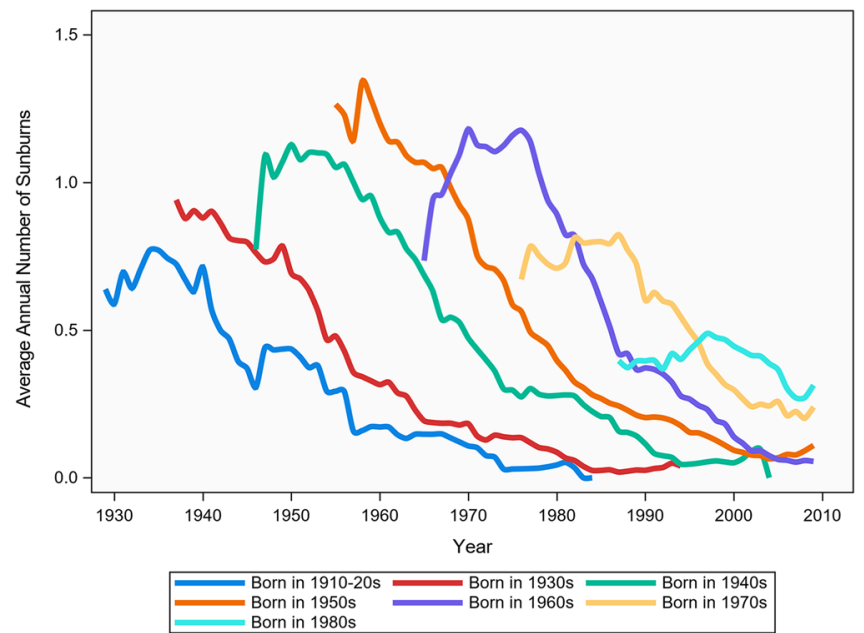

Fig. 1 Trends of average annual sunscreen use $\mathbf{a}$, average annual daily sun exposure (in hours) $\mathbf{b}$, and average annual frequency of sunburns $\mathbf{c}$ over time and by birth cohort among GenoMEL participants 
Table 3 Trends in sunscreen use and sun exposure among GenoMEL participants

\begin{tabular}{|c|c|c|c|c|c|c|c|c|}
\hline \multirow{2}{*}{$\begin{array}{l}\text { Birth } \\
\text { decade }\end{array}$} & \multirow[t]{2}{*}{$n$} & \multirow[t]{2}{*}{ (\%) } & \multicolumn{2}{|c|}{ Sunscreen use } & \multicolumn{4}{|c|}{ Daily sun exposure (hours) } \\
\hline & & & Overall & SPF-Weighted & Overall & During weekdays & During weekends & During holidays \\
\hline \multicolumn{9}{|l|}{ Overall ${ }^{a}$} \\
\hline 1910-1929 & 147 & $(6.4)$ & 2.40 & 2.29 & 2.22 & 2.04 & 2.67 & 1.43 \\
\hline 1930-1939 & 351 & $(15.2)$ & 2.59 & 2.47 & 2.59 & 2.35 & 3.17 & 2.89 \\
\hline 1940-1949 & 493 & $(21.4)$ & 2.57 & 2.39 & 2.52 & 2.19 & 3.32 & 2.73 \\
\hline 1950-1959 & 501 & $(21.7)$ & 2.59 & 2.37 & 2.36 & 1.98 & 3.27 & 2.79 \\
\hline 1960-1969 & 482 & $(20.9)$ & 2.69 & 2.47 & 2.35 & 1.97 & 3.25 & 2.89 \\
\hline 1970-1979 & 292 & $(12.6)$ & 2.87 & 2.55 & 2.45 & 2.13 & 3.19 & 2.82 \\
\hline 1980-1989 & 141 & $(6.1)$ & 2.92 & 2.76 & 2.44 & 2.13 & 3.17 & 2.99 \\
\hline Total & 2407 & & & & & & & \\
\hline Beta $^{b}$ & & & 0.08 & 0.11 & -0.01 & -0.04 & 0.04 & 0.05 \\
\hline$P_{\text {trend }}{ }^{\mathrm{a}}$ & & & 0.004 & 0.0003 & 0.64 & 0.26 & 0.26 & 0.38 \\
\hline \multicolumn{9}{|c|}{10 years old or younger ${ }^{c}$} \\
\hline 1910-1929 & 147 & $(6.4)$ & * & * & 3.40 & 2.38 & 4.30 & 4.80 \\
\hline 1930-1939 & 351 & $(15.2)$ & * & * & 3.48 & 2.42 & 4.61 & 4.83 \\
\hline 1940-1949 & 493 & $(21.4)$ & 1.19 & 1.13 & 3.56 & 2.47 & 4.64 & 4.97 \\
\hline 1950-1959 & 501 & $(21.7)$ & 1.38 & 1.23 & 3.44 & 2.34 & 4.54 & 4.79 \\
\hline 1960-1969 & 482 & (20.9) & 1.89 & 1.61 & 3.42 & 2.39 & 4.46 & 4.66 \\
\hline 1970-1979 & 292 & $(12.6)$ & 2.47 & 2.18 & 3.43 & 2.41 & 4.36 & 4.64 \\
\hline 1980-1989 & 141 & $(6.1)$ & 3.18 & 2.99 & 3.19 & 2.37 & 3.95 & 4.24 \\
\hline Total & 2407 & & & & & & & \\
\hline Beta $^{b}$ & & & 0.41 & 0.34 & -0.03 & -0.01 & -0.07 & -0.08 \\
\hline$P_{\text {trend }}{ }^{c}$ & & & $<0.0001$ & $<0.0001$ & 0.04 & 0.65 & 0.003 & 0.0002 \\
\hline \multicolumn{9}{|c|}{11 to 20 years old ${ }^{c}$} \\
\hline 1910-1929 & 147 & (6.4) & * & * & 2.79 & 2.22 & 3.68 & 3.29 \\
\hline 1930-1939 & 351 & $(15.2)$ & 1.32 & 1.21 & 2.86 & 2.20 & 3.93 & 3.48 \\
\hline 1940-1949 & 493 & (21.4) & 1.37 & 1.23 & 2.88 & 2.14 & 3.92 & 3.83 \\
\hline 1950-1959 & 501 & $(21.7)$ & 1.64 & 1.40 & 2.85 & 2.06 & 3.83 & 3.94 \\
\hline 1960-1969 & 482 & $(20.9)$ & 2.10 & 1.80 & 2.93 & 2.13 & 3.87 & 4.07 \\
\hline 1970-1979 & 292 & $(12.6)$ & 2.83 & 2.42 & 3.01 & 2.26 & 3.79 & 4.05 \\
\hline 1980-1989 & 141 & (6.1) & 3.16 & 2.99 & 2.73 & 2.09 & 3.41 & 3.53 \\
\hline Total & 2407 & & & & & & & \\
\hline $\operatorname{Beta}^{b}$ & & & 0.33 & 0.27 & 0.01 & -0.01 & -0.04 & 0.09 \\
\hline$P_{\text {trend }}{ }^{c}$ & & & $<0.0001$ & $<0.0001$ & 0.37 & 0.76 & 0.04 & 0.0001 \\
\hline \multicolumn{9}{|c|}{ After 20 years old ${ }^{a}$} \\
\hline 1910-1929 & 147 & (6.4) & 2.53 & 2.43 & 2.22 & 2.05 & 2.65 & 1.41 \\
\hline 1930-1939 & 351 & $(15.2)$ & 2.79 & 2.66 & 2.62 & 2.38 & 3.17 & 2.94 \\
\hline 1940-1949 & 493 & $(21.4)$ & 2.98 & 2.78 & 2.53 & 2.21 & 3.30 & 2.78 \\
\hline 1950-1959 & 501 & $(21.7)$ & 3.10 & 2.87 & 2.37 & 1.98 & 3.28 & 2.83 \\
\hline 1960-1969 & 482 & (20.9) & 3.20 & 3.02 & 2.35 & 1.97 & 3.23 & 2.87 \\
\hline 1970-1979 & 292 & $(12.6)$ & 3.30 & 3.05 & 2.49 & 2.17 & 3.17 & 2.74 \\
\hline 1980-1989 & 141 & $(6.1)$ & 2.93 & 2.76 & 2.51 & 2.15 & 3.18 & 2.97 \\
\hline Total & 2407 & & & & & & & \\
\hline Beta $^{b}$ & & & 0.13 & 0.10 & -0.01 & -0.03 & 0.03 & 0.03 \\
\hline
\end{tabular}


Table 3 Trends in sunscreen use and sun exposure among GenoMEL participants (Continued)

\begin{tabular}{|c|c|c|c|c|c|c|c|c|}
\hline \multirow{2}{*}{$\begin{array}{l}\text { Birth } \\
\text { decade }\end{array}$} & \multirow[t]{2}{*}{$n$} & \multirow[t]{2}{*}{ (\%) } & \multicolumn{2}{|c|}{ Sunscreen use } & \multicolumn{4}{|c|}{ Daily sun exposure (hours) } \\
\hline & & & Overall & SPF-Weighted & Overall & During weekdays & During weekends & During holidays \\
\hline$P_{\text {trend }}^{a}$ & & & 0.0002 & 0.002 & 0.83 & 0.31 & 0.33 & 0.64 \\
\hline \multicolumn{9}{|l|}{ Non-cases ${ }^{c}$} \\
\hline 1910-1929 & 77 & $(6.1)$ & 1.71 & 1.60 & 2.59 & 2.50 & 2.84 & 1.39 \\
\hline 1930-1939 & 164 & $(13.0)$ & 2.15 & 2.03 & 2.68 & 2.44 & 3.25 & 2.90 \\
\hline 1940-1949 & 207 & $(16.5)$ & 2.34 & 2.14 & 2.71 & 2.40 & 3.50 & 2.98 \\
\hline 1950-1959 & 268 & (21.3) & 2.38 & 2.13 & 2.50 & 2.14 & 3.36 & 2.98 \\
\hline 1960-1969 & 242 & $(19.3)$ & 2.76 & 2.47 & 2.49 & 2.11 & 3.38 & 3.08 \\
\hline 1970-1979 & 189 & $(15.0)$ & 2.97 & 2.68 & 2.34 & 1.98 & 3.17 & 3.03 \\
\hline 1980-1989 & 110 & (8.8) & 3.26 & 3.11 & 2.48 & 2.15 & 3.24 & 3.21 \\
\hline Total & 1257 & & & & & & & \\
\hline $\operatorname{Beta}^{b}$ & & & 0.23 & 0.24 & -0.06 & -0.09 & 0.01 & 0.08 \\
\hline$P_{\text {trend }}{ }^{c}$ & & & $<0.0001$ & $<0.0001$ & 0.02 & 0.001 & 0.73 & 0.10 \\
\hline \multicolumn{9}{|c|}{ Melanoma Cases ${ }^{a}$} \\
\hline 1910-1929 & 70 & $(6.1)$ & 2.70 & 2.57 & 2.04 & 1.81 & 2.59 & 1.82 \\
\hline 1930-1939 & 187 & (16.3) & 2.69 & 2.55 & 2.63 & 2.41 & 3.15 & 3.10 \\
\hline 1940-1949 & 286 & (24.9) & 2.64 & 2.46 & 2.39 & 2.08 & 3.18 & 2.63 \\
\hline 1950-1959 & 233 & (20.3) & 2.83 & 2.65 & 2.20 & 1.80 & 3.16 & 2.57 \\
\hline 1960-1969 & 240 & (20.9) & 2.75 & 2.59 & 2.13 & 1.71 & 3.09 & 2.59 \\
\hline 1970-1979 & 103 & (9.0) & 3.13 & 2.74 & 2.52 & 2.23 & 3.22 & 2.44 \\
\hline 1980-1989 & 31 & $(2.7)$ & 2.92 & 2.71 & 1.91 & 1.47 & 2.94 & 2.45 \\
\hline Total & 1150 & & & & & & & \\
\hline Beta $^{b}$ & & & 0.07 & 0.10 & -0.04 & -0.07 & 0.04 & -0.07 \\
\hline$P_{\text {trend }}{ }^{\mathrm{a}}$ & & & 0.08 & 0.02 & 0.33 & 0.11 & 0.25 & 0.43 \\
\hline Adjusted Mean & fference ${ }^{a}$ & & 0.008 & 0.06 & -0.11 & -0.12 & -0.07 & -0.46 \\
\hline$P_{A M D}{ }^{a}$ & & & 0.91 & 0.36 & 0.11 & 0.15 & 0.39 & 0.002 \\
\hline$P_{\text {heterogeneity of tr }}$ & & & 0.69 & 0.69 & 0.59 & 0.85 & 0.31 & 0.51 \\
\hline \multicolumn{9}{|l|}{ Pre-Diagnosis ${ }^{c}$} \\
\hline 1910-1929 & 70 & $(6.1)$ & 2.10 & 1.94 & 2.31 & 1.84 & 3.07 & 3.58 \\
\hline 1930-1939 & 187 & $(16.3)$ & 2.10 & 1.92 & 2.83 & 2.42 & 3.52 & 3.78 \\
\hline 1940-1949 & 285 & (24.9) & 2.07 & 1.85 & 2.70 & 2.12 & 3.63 & 3.98 \\
\hline 1950-1959 & 232 & (20.3) & 2.35 & 2.14 & 2.64 & 1.98 & 3.58 & 3.88 \\
\hline 1960-1969 & 239 & (20.9) & 2.50 & 2.33 & 2.72 & 1.98 & 3.65 & 3.96 \\
\hline 1970-1979 & 101 & (8.8) & 3.15 & 2.71 & 3.02 & 2.26 & 3.73 & 4.13 \\
\hline 1980-1989 & 31 & $(2.7)$ & 3.11 & 2.86 & 2.78 & 2.05 & 3.57 & 3.75 \\
\hline Total & 1145 & & & & & & & \\
\hline Beta $^{b}$ & & & 0.18 & 0.16 & 0.05 & -0.03 & 0.06 & 0.05 \\
\hline$P_{\text {trend }}{ }^{c}$ & & & $<0.0001$ & $<0.0001$ & 0.02 & 0.23 & 0.01 & 0.07 \\
\hline \multicolumn{9}{|c|}{ Post-Diagnosis $^{c}$} \\
\hline 1910-1929 & 70 & $(6.1)$ & 2.79 & 2.73 & 1.92 & 1.86 & 2.11 & 2.48 \\
\hline 1930-1939 & 186 & (16.4) & 3.16 & 3.07 & 2.62 & 2.53 & 2.86 & 3.43 \\
\hline 1940-1949 & 283 & $(25.0)$ & 3.71 & 3.64 & 2.42 & 2.20 & 2.93 & 2.74 \\
\hline 1950-1959 & 231 & (20.4) & 4.08 & 4.02 & 2.10 & 1.68 & 3.05 & 3.08 \\
\hline 1960-1969 & 234 & (20.6) & 3.91 & 3.87 & 2.08 & 1.70 & 2.98 & 2.78 \\
\hline
\end{tabular}


Table 3 Trends in sunscreen use and sun exposure among GenoMEL participants (Continued)

\begin{tabular}{|c|c|c|c|c|c|c|c|c|}
\hline \multirow{2}{*}{$\begin{array}{l}\text { Birth } \\
\text { decade }\end{array}$} & \multirow[t]{2}{*}{$n$} & \multirow[t]{2}{*}{ (\%) } & \multicolumn{2}{|c|}{ Sunscreen use } & \multicolumn{4}{|c|}{ Daily sun exposure (hours) } \\
\hline & & & Overall & SPF-Weighted & Overall & During weekdays & During weekends & During holidays \\
\hline 1970-1979 & 101 & (8.9) & 4.04 & 3.91 & 2.63 & 2.31 & 3.33 & 3.54 \\
\hline 1980-1989 & 29 & $(2.6)$ & 3.47 & 3.40 & 2.28 & 1.77 & 3.23 & 3.09 \\
\hline Total & 1134 & & & & & & & \\
\hline \multicolumn{3}{|l|}{ Beta $^{b}$} & 0.24 & 0.24 & -0.02 & -0.10 & 0.13 & 0.04 \\
\hline \multicolumn{3}{|l|}{$P_{\text {trend }}^{c}$} & $<0.0001$ & $<0.0001$ & 0.43 & 0.003 & $<0.0001$ & 0.62 \\
\hline \multicolumn{3}{|c|}{ Adjusted Mean Difference ${ }^{d}$} & 1.36 & 1.5 & -0.41 & -0.08 & -0.63 & -0.88 \\
\hline \multicolumn{3}{|l|}{$P_{A M D}{ }^{d}$} & $<0.0001$ & $<0.0001$ & $<0.0001$ & 0.04 & $<0.0001$ & $<0.0001$ \\
\hline \multicolumn{3}{|c|}{$P_{\text {heterogeneity of trend }}{ }^{d}$} & 0.04 & 0.001 & 0.10 & $<0.0001$ & 0.0004 & 0.94 \\
\hline
\end{tabular}

* Sunscreen was not widely available until the 1950s; analyses were limited to 1950s and afterwards

a Model adjusted for sex, phenotype factor variables, center geography, years unaffected, and clustered by family

${ }^{\mathrm{b}}$ Betas represent the average change per unit increase in birth decade, after adjusting for covariates

' Model adjusted for sex, phenotype factor variables, center geography, and clustered by family

${ }^{\mathrm{d}}$ Model adjusted for repeated individual data clustered by family

\section{Sun exposure}

There was no apparent difference in unadjusted average sun exposure across birth cohorts or over time period, but sun exposure decreased as each cohort aged (Fig. 1b). We did not observe a trend in the adjusted lifetime average daily sun exposure with increasing birth cohort overall, or during weekdays, weekends, or holidays (Table 3$)$. At 10 years of age or younger, average daily sun exposure during weekends $\left(\beta=-0.07, P_{\text {trend }}=\right.$ $0.003)$, holidays $\left(\beta=-0.08, P_{\text {trend }}=0.0002\right)$, and overall $\left(\beta=-0.03, P_{\text {trend }}=0.04\right)$ decreased in more recent birth cohorts. This trend remained for weekends $(\beta=-0.07$, $\left.P_{\text {trend }}=0.04\right)$, although sun exposure increased during holidays $\left(\beta=0.09, P_{\text {trend }}=0.0001\right)$ when participants were between 11 to 20 years old. We did not observe a trend in average sun exposure by birth cohort when participants were older than 20 years. Cases had less sun exposure during holidays $(\mathrm{AMD}=-0.46 \mathrm{~h}, \quad P=0.002)$ compared to non-cases, otherwise there were no differences or heterogeneity in trend for sun exposure between cases and non-cases.

After diagnosis, cases had lower average daily sun exposure overall (AMD $=-0.41 \mathrm{~h}, P<0.0001)$, which was primarily driven by differences during weekends (AMD $=-0.63 \mathrm{~h}, P<0.0001)$, holidays $(\mathrm{AMD}=-0.88 \mathrm{~h}$, $P<0.0001$ ), and to a lesser extent, weekdays (AMD =$0.08 \mathrm{~h}, P=0.04$ ), compared to before diagnosis. Although there was no heterogeneity of trend overall and during holidays, there was an accelerated decrease across the birth cohorts in sun exposure during weekdays $(P<$ $0.0001)$ and weekends $(P=0.0004)$ after diagnosis compared to before diagnosis.

\section{Sunburns}

Within birth cohorts, average number of sunburns occurred more frequently at younger ages and decreased with age (Fig. 1c). The frequency of early life sunburns increased until the 1950 s birth cohort and then decreased, with the lowest frequency of sunburns at younger ages observed in the most recent (1980s) cohort. Similarly, frequency of sunburns increased and peaked in 1960 and decreased thereafter.

A total of 1598 (66.4\%) respondents reported ever being sunburned (Table 4). We did not observe a trend of odds of ever being sunburned with increasing birth cohort or within the three life periods after adjustment. Cohort-specific odds ratios tended to increase until the 1950 s cohort and decrease thereafter. This inverted Ushape trend was verified in models containing a quadratic birth cohort term (data not shown). There was no difference in odds of ever being sunburned between melanoma cases and non-cases $(\mathrm{OR}=1.12,95 \% \mathrm{CI}$ : 0.88 to 1.42), nor was there heterogeneity of trend. Cases were less likely to have sunburns after diagnosis with melanoma than before $(\mathrm{OR}=0.04,95 \% \mathrm{CI}$ : 0.04 to 0.05$)$. The trend in odds of ever being sunburned across subsequent birth cohorts was (inverted) U-shaped before diagnosis, in contrast to an increasing linear trend seen after diagnosis.

\section{Sunbed use}

Less than a third $(n=683,28.6 \%)$ of respondents reported ever using sunbeds (Table 4). There was a trend of increasing odds of sunbed use with increasing birth cohort (per cohort OR $=1.44,95 \% \mathrm{CI}: 1.28$ to 1.62), although the odds ratio estimate for the 1980s cohort was less than the 1970s cohort. A similar trend was seen after stratifying by melanoma status. There was no difference in odds of sunbed use or heterogeneity in trend when comparing cases and non-cases. Cases were less likely to use sunbeds after diagnosis than before diagnosis $(\mathrm{OR}=0.19,95 \% \mathrm{CI}$ : 0.14 to 0.25$)$. The trend in odds ratios of sunbed use by birth cohort before diagnosis was similar to that 
Table 4 Adjusted odds ratios and 95\% confidence intervals for the association between birth decade and history of sunburns and sunbed use among GenoMEL participants

\begin{tabular}{|c|c|c|c|c|c|c|c|c|c|c|}
\hline \multirow[t]{2}{*}{ Birth decade } & \multicolumn{5}{|c|}{ Sunburn } & \multicolumn{5}{|c|}{ Sunbed use } \\
\hline & $n$ & Never $n(\%)$ & Ever $n(\%)$ & OR & $95 \% \mathrm{Cl}$ & $n$ & Never $n(\%)$ & Ever $n(\%)$ & OR & $95 \% \mathrm{Cl}$ \\
\hline \multicolumn{11}{|l|}{ Overall ${ }^{a}$} \\
\hline 1910-1929 & 147 & $63(42.9)$ & $84(57.1)$ & 1.00 & REF & 144 & $130(90.3)$ & $14(9.7)$ & 1.00 & REF \\
\hline 1930-1939 & 351 & 147 (41.9) & $204(58.1)$ & 1.07 & (0.70 1.63) & 349 & $292(83.7)$ & $57(16.3)$ & & \\
\hline 1940-1949 & 493 & $142(28.8)$ & 351 (71.2) & 2.03 & (1.28 3.22) & 492 & $366(74.4)$ & $126(25.6)$ & 2.29 & (1.48 3.53) \\
\hline 1950-1959 & 501 & $132(26.4)$ & $369(73.7)$ & 2.20 & (1.39 3.48) & 497 & $353(71.0)$ & $144(29.0)$ & 3.05 & (1.94 4.79) \\
\hline 1960-1969 & 482 & $137(28.4)$ & $345(71.6)$ & 1.92 & (1.10 3.35) & 476 & $294(61.8)$ & $182(38.2)$ & 6.12 & (3.78 9.89) \\
\hline 1970-1979 & 292 & $113(38.7)$ & 179 (61.3) & 1.12 & (0.60 2.11) & 289 & $173(59.9)$ & $116(40.1)$ & 6.36 & (3.53 11.44) \\
\hline 1980-1989 & 141 & $75(53.2)$ & $66(46.8)$ & 0.69 & (0.32 1.46) & 138 & $94(68.1)$ & $44(31.9)$ & 4.38 & (2.12 9.07) \\
\hline Total & 2407 & 809 (33.6) & $1598(66.4)$ & & & 2385 & $1702(71.4)$ & $683(28.6)$ & & \\
\hline Per Cohort OR & & & & 0.96 & (0.86 1.08) & & & & 1.44 & (1.28 1.62) \\
\hline$P_{\text {trend }}$ & & & & 0.52 & & & & & $<0.0001$ & \\
\hline \multicolumn{11}{|c|}{10 years old or younger ${ }^{b}$} \\
\hline 1910-1929 & 140 & $93(66.4)$ & 47 (33.6) & 1.00 & REF & * & & & & \\
\hline 1930-1939 & 331 & $212(64.0)$ & $119(36.0)$ & 1.24 & (0.78 1.99) & * & & & & \\
\hline 1940-1949 & 467 & $270(57.8)$ & $197(42.2)$ & 1.68 & (1.07 2.62) & * & & & & \\
\hline 1950-1959 & 481 & $253(52.6)$ & $228(47.4)$ & 2.05 & (1.32 3.17) & * & & & & \\
\hline 1960-1969 & 464 & $276(59.5)$ & $188(40.5)$ & 1.57 & $(0.972 .54)$ & * & & & & \\
\hline 1970-1979 & 283 & $191(67.5)$ & $92(32.5)$ & 1.04 & $(0.621 .73)$ & * & & & & \\
\hline 1980-1989 & 139 & $108(77.7)$ & $31(22.3)$ & 0.71 & (0.39 1.29) & * & & & & \\
\hline Total & 2305 & $1403(58.3)$ & $902(41.7)$ & & & & & & & \\
\hline Per Cohort OR & & & & 0.96 & $(0.901 .02)$ & & & & & \\
\hline$P_{\text {trend }}$ & & & & 0.21 & & & & & & \\
\hline \multicolumn{11}{|c|}{11 to 20 years old $b$} \\
\hline 1910-1929 & 147 & $68(46.3)$ & $79(53.7)$ & 1.00 & REF & * & & & & \\
\hline 1930-1939 & 349 & $160(45.9)$ & $189(54.1)$ & 1.07 & $(0.711 .61)$ & * & & & & \\
\hline 1940-1949 & 493 & $165(33.5)$ & $328(66.5)$ & 1.88 & $(1.242 .85)$ & * & & & & \\
\hline 1950-1959 & 501 & $155(30.9)$ & $346(69.1)$ & 2.10 & (1.42 3.12) & * & & & & \\
\hline 1960-1969 & 479 & $146(30.5)$ & $333(69.5)$ & 2.27 & $(1.473 .51)$ & * & & & & \\
\hline 1970-1979 & 292 & $119(40.8)$ & $173(59.2)$ & 1.36 & (0.87 2.13) & * & & & & \\
\hline 1980-1989 & 141 & $76(53.9)$ & $65(46.1)$ & 0.94 & $(0.561 .57)$ & * & & & & \\
\hline Total & 2402 & $889(36.9)$ & $1513(63.1)$ & & & & & & & \\
\hline Per Cohort OR & & & & 1.04 & (0.97 1.11) & & & & & \\
\hline$P_{\text {trend }}$ & & & & 0.30 & & & & & & \\
\hline \multicolumn{11}{|c|}{ After 20 years old ${ }^{a}$} \\
\hline 1910-1929 & 147 & $114(77.6)$ & $33(22.4)$ & 1.00 & REF & & & & & \\
\hline 1930-1939 & 351 & $242(69.0)$ & $109(31.0)$ & 1.71 & (1.04 2.83) & * & & & & \\
\hline 1940-1949 & 491 & $278(56.6)$ & $213(43.4)$ & 3.13 & (1.89 5.20) & * & & & & \\
\hline 1950-1959 & 501 & $283(56.5)$ & $218(43.5)$ & 3.29 & (1.97 5.49) & * & & & & \\
\hline 1960-1969 & 482 & $297(61.6)$ & $185(38.4)$ & 2.82 & (1.60 4.96) & * & & & & \\
\hline 1970-1979 & 291 & $197(67.7)$ & $94(32.3)$ & 2.31 & $(1.244 .29)$ & * & & & & \\
\hline 1980-1989 & 106 & $82(77.4)$ & $24(22.6)$ & 1.47 & $(0.673 .22)$ & * & & & & \\
\hline Total & 2369 & $1493(63.0)$ & 876 (37.0) & & & * & & & & \\
\hline
\end{tabular}


Table 4 Adjusted odds ratios and 95\% confidence intervals for the association between birth decade and history of sunburns and sunbed use among GenoMEL participants (Continued)

\begin{tabular}{|c|c|c|c|c|c|c|c|c|c|c|}
\hline \multirow[t]{2}{*}{ Birth decade } & \multicolumn{5}{|c|}{ Sunburn } & \multicolumn{5}{|c|}{ Sunbed use } \\
\hline & $n$ & Never $n(\%)$ & Ever $n(\%)$ & OR & $95 \% \mathrm{Cl}$ & $n$ & Never $\boldsymbol{n}(\%)$ & Ever $n(\%)$ & OR & $95 \% \mathrm{Cl}$ \\
\hline Per Cohort OR & & & & 1.05 & (0.96 1.14) & & & & & \\
\hline$P_{\text {trend }}$ & & & & 0.33 & & & & & & \\
\hline \multicolumn{11}{|l|}{ Non-cases ${ }^{b}$} \\
\hline 1910-1929 & 77 & $36(46.8)$ & $41(53.3)$ & 1.00 & REF & 74 & $67(90.5)$ & $7(9.5)$ & 1.00 & REF \\
\hline 1930-1939 & 164 & $71(43.3)$ & $93(56.7)$ & 1.08 & (0.60 1.95) & 163 & 199 (84.0) & 39 (16.0) & & \\
\hline 1940-1949 & 207 & 78 (37.7) & $129(62.3)$ & 1.64 & (0.94 2.86) & 207 & $141(68.1)$ & 66 (31.9) & 2.06 & (1.19 3.55) \\
\hline 1950-1959 & 268 & 74 (27.6) & $194(72.4)$ & 2.60 & $(1.474 .60)$ & 266 & $178(66.9)$ & 88 (33.1) & 2.11 & $(1.203 .55)$ \\
\hline 1960-1969 & 242 & $80(33.1)$ & $162(66.9)$ & 2.06 & $(1.173 .65)$ & 240 & $145(60.4)$ & 95 (39.6) & 3.20 & $(1.895 .41)$ \\
\hline 1970-1979 & 189 & $81(42.9)$ & 108 (57.1) & 1.19 & (0.65 2.15) & 189 & 110 (58.2) & 79 (41.8) & 3.20 & $(1.815 .63)$ \\
\hline 1980-1989 & 110 & $60(54.6)$ & $50(45.4)$ & 0.86 & (0.44 1.68) & 108 & 72 (66.7) & $36(33.3)$ & 2.03 & (1.09 3.77) \\
\hline Total & 1257 & $480(38.2)$ & 777 (61.8) & & & 1247 & $845(67.8)$ & $402(32.2)$ & & \\
\hline Per Cohort OR & & & & 0.99 & (0.91 1.08) & & & & 1.19 & (1.09 1.30) \\
\hline$P_{\text {trend }}$ & & & & 0.78 & & & & & 0.0002 & \\
\hline \multicolumn{11}{|l|}{ Melanoma cases $^{a}$} \\
\hline 1910-1929 & 70 & 27 (38.6) & $43(61.4)$ & 1.00 & REF & 70 & $63(90.0)$ & $7(10.0))$ & 1.00 & REF \\
\hline 1930-1939 & 187 & 76 (40.6) & $111(59.4)$ & 1.10 & $(0.58$ 2.11) & 186 & 160 (86.0) & $26(14.0))$ & & \\
\hline 1940-1949 & 286 & $64(22.4)$ & $222(77.6)$ & 2.90 & (1.48 5.66) & 285 & $225(79.0)$ & $60(21.0)$ & 2.06 & (1.17 3.64) \\
\hline 1950-1959 & 233 & $58(24.9)$ & $175(75.1)$ & 2.44 & (1.27 4.70) & 231 & 175 (75.8) & $56(24.2)$ & 2.73 & (1.47 5.04) \\
\hline 1960-1969 & 240 & $57(23.8)$ & $183(76.2)$ & 2.68 & (1.21 5.93) & 236 & 149 (63.1) & 87 (36.9) & 6.46 & (3.47 12.05) \\
\hline 1970-1979 & 103 & $32(31.1)$ & 71 (68.9) & 1.89 & $(0.794 .50)$ & 100 & $63(63.0)$ & $37(37.0)$ & 5.80 & (2.60 12.93) \\
\hline 1980-1989 & 31 & $15(48.4)$ & $16(51.6)$ & 0.92 & $(0.292 .92)$ & 30 & $22(73.3)$ & $8(26.7)$ & 2.83 & (0.81 9.94) \\
\hline Total & 1150 & 329 (28.6) & $821(71.4)$ & & & 1138 & 857 (75.3) & $281(24.7)$ & & \\
\hline Per Cohort OR & & & & 1.00 & $(0.99$ 1.02) & & & & 1.46 & $(1.24$ 1.71) \\
\hline$P_{\text {trend }}$ & & & & 0.29 & & & & & $<0.0001$ & \\
\hline$O R_{\text {cases vs non-cases }}$ & & & & 1.12 & $(0.88$ 1.42) & & & & 0.89 & (0.67 1.16) \\
\hline$P_{\text {heterogeneity of trend }}$ & & & & 0.21 & & & & & 0.21 & \\
\hline \multicolumn{11}{|l|}{ Pre-Diagnosis $^{b}$} \\
\hline 1910-1929 & 70 & 27 (38.6) & $43(61.4)$ & 1.00 & REF & 70 & $65(92.9)$ & $5(7.1)$ & 1.00 & REF \\
\hline 1930-1939 & 187 & $77(41.2)$ & $110(58.8)$ & & & 183 & $163(89.1)$ & $20(10.9)$ & & \\
\hline 1940-1949 & 285 & $63(22.1)$ & $222(77.9)$ & 2.62 & (1.69 4.05) & 285 & $237(83.2)$ & $48(16.8)$ & 1.86 & (1.05 3.29) \\
\hline 1950-1959 & 232 & $57(24.6)$ & $175(75.4)$ & 2.16 & (1.44 3.25) & 226 & $182(80.5)$ & 44 (19.5) & 2.26 & $(1.25$ 4.07) \\
\hline 1960-1969 & 238 & $56(23.5)$ & $182(76.5)$ & 2.38 & $(1.503 .77)$ & 234 & $156(66.7)$ & 78 (33.3) & 5.45 & (3.26 9.10) \\
\hline 1970-1979 & 101 & $31(30.7)$ & 70 (69.3) & 1.68 & (0.96 2.92) & 99 & $64(64.7)$ & 35 (35.3) & 4.98 & (2.63 9.43) \\
\hline 1980-1989 & 31 & 15 (48.4) & $16(51.6)$ & 0.77 & (0.34 1.78) & 30 & $23(76.7)$ & $7(23.3)$ & 2.05 & $(0.646 .58)$ \\
\hline Total & 1144 & $326(28.5)$ & 818 (71.5) & & & 1127 & $890(79.0)$ & $237(21.0)$ & & \\
\hline Per Cohort OR & & & & 1.09 & $(0.98$ 1.21) & & & & 1.41 & $\left(\begin{array}{lll}1.25 & 1.59\end{array}\right)$ \\
\hline$P_{\text {trend }}$ & & & & 0.12 & & & & & $<0.0001$ & \\
\hline \multicolumn{11}{|l|}{ Post-Diagnosis $^{b}$} \\
\hline 1910-1929 & 68 & 66 (97.1) & $2(2.9)$ & 1.00 & REF & 70 & $68(97.1)$ & $2(2.9)$ & 1.00 & REF \\
\hline 1930-1939 & 184 & $176(95.7)$ & $8(4.3)$ & & & 187 & 181 (96.8) & $6(3.2)$ & & \\
\hline 1940-1949 & 281 & $265(94.3)$ & $16(5.7)$ & 2.08 & $(0.835 .26)$ & 286 & $270(94.4)$ & $16(5.6)$ & 1.57 & (0.64 3.88) \\
\hline 1950-1959 & 226 & 199 (88.1) & $27(11.9)$ & 4.40 & (1.88 10.29) & 233 & 222 (95.3) & $11(4.7)$ & 1.33 & $(0.463 .81)$ \\
\hline
\end{tabular}


Table 4 Adjusted odds ratios and 95\% confidence intervals for the association between birth decade and history of sunburns and sunbed use among GenoMEL participants (Continued)

\begin{tabular}{|c|c|c|c|c|c|c|c|c|c|c|}
\hline \multirow[t]{2}{*}{ Birth decade } & \multicolumn{5}{|c|}{ Sunburn } & \multicolumn{5}{|c|}{ Sunbed use } \\
\hline & $n$ & Never $\boldsymbol{n}(\%)$ & Ever $n(\%)$ & OR & $95 \% \mathrm{Cl}$ & $n$ & Never $\boldsymbol{n}(\%)$ & Ever $n(\%)$ & OR & $95 \% \mathrm{Cl}$ \\
\hline 1960-1969 & 224 & $189(84.4)$ & 35 (15.6) & 6.13 & $\left(\begin{array}{ll}2.60 & 14.43\end{array}\right)$ & 240 & $228(95.0)$ & $12(5.0)$ & 1.34 & $(0.593 .04)$ \\
\hline 1970-1979 & 95 & $81(85.3)$ & $14(14.7)$ & 5.80 & (2.20 15.27) & 102 & $97(95.1)$ & $5(4.9)$ & 1.24 & $(0.384 .00)$ \\
\hline 1980-1989 & 27 & $20(74.1)$ & $7(25.9)$ & 10.53 & (3.24 34.29) & 31 & 29 (93.6) & $2(6.5)$ & 1.61 & $(0.308 .60)$ \\
\hline Total & 1105 & $996(90.1)$ & $109(9.9)$ & & & 1149 & 1095 (95.3) & $54(4.7)$ & & \\
\hline Per Cohort OR & & & & 1.53 & (1.32 1.76) & & & & 1.03 & (0.85 1.25) \\
\hline$P_{\text {trend }}$ & & & & $<0.0001$ & & & & & 0.76 & \\
\hline$O R_{\text {post vs pre }}{ }^{c}$ & & & & 0.04 & (0.04 0.05) & & & & 0.19 & (0.14 0.25) \\
\hline$P_{\text {heterogeneity of trend }}{ }^{c}$ & & & & $<0.0001$ & & & & & 0.008 & \\
\hline
\end{tabular}

* Participants were only asked about ever use of sunbeds once and were not asked to recall sunbed use at anchor ages

${ }^{a}$ Model adjusted for sex, phenotype factor variables, center geography, years unaffected and clustered by family

${ }^{\mathrm{b}}$ Model adjusted for sex, phenotype factor variables, center geography, and clustered by family

c Model adjusted for repeated individual data clustered by family

observed overall (per cohort OR $=1.41,95 \% \mathrm{CI}: 1.25$ to 1.59), while there was no trend in sunbed use by birth cohort after diagnosis.

\section{Discussion}

To our knowledge, this is the first study to investigate trends of sun-related behavior by birth cohort among individuals in melanoma-prone families. We found increased sunscreen use with increasing birth cohort, regardless of SPF; and although we saw a trend of increasing odds of sunburn until the 1950s birth cohort, it decreased thereafter. We found no trends of sun exposure across birth cohorts, but we did note a trend of increasing odds of sunbed use with increasing birth cohort.

In our analysis of birth cohort-specific trends in sunrelated behaviors by life period, we found that most participants experienced a sunburn at 11 to 20 years old, a time frame in which we also saw a trend of increasing sun exposure with increasing birth cohort during holidays. Sunscreen use was also lower during childhood (10 years old or younger) and adolescence (11 to 20 years old) compared to adulthood (after 20 years old). Because childhood and adolescence are critical windows of exposure for melanoma risk [16, 17], future interventions should be targeted towards these age groups.

When comparing between melanoma cases and noncases, we found no differences in sunscreen use, sunburns, sunbed use, and sun exposure overall, during weekdays and weekends. A previous study also found no differences in lifetime or childhood sun exposure between cases and non-cases from melanoma-prone families [32]. These similarities may be due to shared behavior within families. However, we observed that cases had greater average sun exposure during holidays than non-cases, which may support the importance of intermittent sun exposure in melanoma pathogenesis within melanoma-prone families. Future research may need to focus on measures of intermittent sun exposure, such as the frequency and duration of specific recreational and vacation activities [33], as drivers of individual melanoma risk among melanoma-prone families.

Previous studies reported that a personal melanoma diagnosis acts as the strongest catalyst in changing one's sun-related behavior [10,34, 35]. In our study, we found higher sunscreen use, lower sun exposure, lower odds of sunburn and sunbed use among cases after diagnosis than before. However, we found a trend of increasing risk of sunburn with increasing birth cohort after diagnosis - a possible target for further investigation and prevention strategies.

Although we observed increased sunbed use in more recent birth cohorts compared to older ones, sunbeds and indoor tanning have become increasingly regulated by governments, including outright bans and restricted use by minors $[36,37]$. In the last two decades, sunbed use has trended downwards among adolescents and adults globally [36, 38, 39].

We did not ask participants about their beliefs and/or attitudes regarding sun-related behaviors in our sunexposure questionnaire instrument. A preference for tanned skin has been shown to increase sun-seeking behavior and sunbed use, even among high-risk individuals [10]. We did collect information on whether participants used sunscreen to prolong their sun exposure (data not shown), and although $40 \%$ who used sunscreen reportedly never used it to prolong their sun exposure, onethird $(33 \%)$ of individuals used sunscreen to prolong their sun exposure at least half of the time or more throughout their life. Although we did not collect information on perceived melanoma risk, a subset of GenoMEL participants $(n=312)$ previously completed an 
online survey in which $79 \%$ perceived a greater risk of developing melanoma (or another melanoma) compared to other people of the same sex, age and skin color [40]. Translating this perception into agency remains a challenge.

Limitations of our study include our inability to examine the number of sunburns and number of sunbed sessions as continuous outcomes in our analyses. Both variables were zero-inflated and highly skewed, and our attempts at more complex modeling resulted in nonconvergence due to small sample sizes.

\section{Conclusions}

Although our study and others have shown improvements in sun-protective behavior over time, melanoma primary prevention activities remain suboptimal with room for enhancement during childhood and adolescence. More strategies need to be devised to mitigate melanoma risk by encouraging increased sunscreen use, decreased sun exposure and sunbed use among individuals from melanoma-prone families, with consideration for targeted strategies for individuals younger than 20 years of age.

\section{Supplementary Information}

The online version contains supplementary material available at https://doi. org/10.1186/s12889-021-10424-5.

Additional file 1 : Module 1. Residency calendar, demographics, phenotype, and history of melanoma.

Additional file $\mathbf{2}$ : Module 2. Personal sun exposure

\section{Abbreviations}

AMD: Adjusted mean difference; Cl: Confidence interval; OR: Odds ratio; SD: Standard deviation; SPF: Sun protection factor

\section{Acknowledgements}

We acknowledge the contributions of the participants, their families and the many clinicians, geneticists, genetic counsellors and allied health professionals involved in their management. This work was performed in participation with members of the following study centers:

Barcelona, ES: Paula Aguilera, Llúcia Alós, Celia Badenas, Alicia Barreiro, Neus Calbet, Cristina Carrera, Carlos Conill, Mireia Domínguez, Daniel Gabriel, Pablo Iglesias, Josep Malvehy, M. Eugenia Moliner, Javiera Pérez, Ramon Pigem, Miriam Potrony, Joan Anton Puig Butille, Ramon Rull, Marcelo Sánchez, Gemma Tell-Martí, Sergi Vidal-Sicart, and Oriol Yelamos.

Genoa, IT: Giovanna Bianchi-Scarrà, Lorenza Pastorino, Virginia Andreotti, Claudia Martinuzzi, Bruna Dalmasso, Giulia Ciccarese, Francesco Spagnolo, and Paola Queirolo.

Leeds, UK: Linda Whitaker, Paul Affleck, Jennifer H. Barrett, Jane Harrison, Mark M. Iles, Juliette Randerson-Moor, John C. Taylor, Kairen Kukalizch, Susan Leake, Birute Karpavicius, Sue Haynes, Tricia Mack, May Chan, and Yvonne Taylor.

Leiden, NL: Clasine van der Drift, Leny van Mourik, Wilma Bergman, Femke de Snoo, Jeanet ter Huurne, and Frans van Nieuwpoort.

Lund. SE: wishes to thank Anita Zander, R.N. for invaluable help with the data from the Lund Melanoma Study Group and acknowledge Kari Nielsen, Anna Måsbäck, Katja Harbst, Goran Jonsson and Åke Borg.

Mexico, MX: the authors thank Victoria Godinez Puig from Mexico for helping to collect patient samples and data.

Montevideo, UY: Virginia Barquet, Javiera Pérez, Miguel Martínez, Jimena Núñez, and Malena Scarone.
Philadelphia, PA, US (University of Pennsylvania): acknowledges the contributions of Patricia Van Belle, Althea Ruffin, Jillian Knorr, and Wenting Zhou.

Porto Alegre, BR: Renan Rangel Bonamigo and Maria Carolina Widholzer Rey.

Riga, LV: Kristine Azarjana, Simona Donina, Olita Heisele, Baiba Štreinerte, Aija Ozola and Ludmila Engele.

São Paulo, BR: Dirce Maria Carraro, Alexandre Leon Ribeiro de Ávila, Bianca Costa Soares de Sá, Maria Isabel Waddington Achatz, and João Duprat. Throughout data collection, Gilles Landman was affiliated with the AC Camargo Cancer Center, São Paulo, Brazil.

Stockholm, SE: wishes to thank Diana Lindén, R.N. for excellent work collecting and entering data into the study data base and Rainer Tuominen for screening of CDKN2A.

Sydney, AU: Caroline Watts, Gayathri St. George, Robyn Dalziell and Kate McBride who assisted with recruitment of study participants; Leo Raudonikis who assisted with data management; and Chantelle Agha-Hamilton and Svetlana Pianova who assisted with biospecimen management.

Tel Aviv, IL: wishes to acknowledge Esther Azizi, Yael Laitman.

\section{Authors' contributions}

PAK and LARF conceived the study. Data were curated by LQ and SHD. Formal Analyses were performed by JCAL, SAZ, NM, LQ, SHD, and PAK Funding was acquired by DTB, DEE, JANB, and PAK. Methodology was devised by JCAL, SAZ, LARF, NM and PAK. Recruitment, data collection, and management of participants were done by EA, CB, DTB, WB, BCO, FC, AEC, DEE, AMG, PG, TCG, NAG, JH, MH, VH, EAH, Cl, GL, ALB, GJM, MM, LFM, HO, JJOL, BP, DP, SP, JSA, HS, KAWW, JANB, and PAK. The manuscript was drafted by JCAL, SAZ, LARF, and PAK. The author(s) read and approved the final manuscript.

\section{Funding}

This work was supported by: the European Commission under the 6th and 7th Framework Programme [LSH-CT-2006-018702] to JNB; Cancer Research UK Programme Awards (C588/A4994 and C588/ A10589) to DTB/JNB; a Cancer Research UK Project Grant (C8216/A6129) to DTB/JNB; the US National Institutes of Health [R01 CA83115 (PAK, DEE, DTB)], the National Health and Medical Research Council of Australia [NHMRC 107359, 402761, 633004, 566946, 211172, 1093017, 1147843 (AC)]; the Cancer Council New South Wales (project grant 77/00, 06/10); the Cancer Institute New South Wales [CINSW 05/TPG/1-01, 10/TPG/1-02], 15/CDF/1-14 (AC); CAPES (Coordenação de Aperfeiçoamento de Pessoal de Nível Superior); FAPESP (Fundação para o Amparo da Pesquisa do Estado de São Paulo) - SP, Brazil \# 2007/04313-2; the Cancer Research Foundations of Radiumhemmet and the Swedish Cancer Society; the Paulsson Trust, Lund University; grant support from the Swedish Cancer Society and European Research Council Advanced Grant (ERC-2011-294576); the research at the Melanoma Unit in Barcelona is partially funded by Spanish Fondo de Investigaciones Sanitarias grants PI15/ 00716 and PI15/00956; CIBER de Enfermedades Raras of the Instituto de Salud Carlos III, Spain, co-financed by European Regional Development Fund "A way to achieve Europe" ERDF; AGAUR 2014_SGR_603 of the Catalan Government, Spain; Diagnoptics; a grant from "Fundació La Marató de TV3" 201331-30, Catalonia, Spain; a grant from "Fundación Científica de la Asociación Española Contra el Cáncer" GCB15152978SOEN, Spain, and CERCA Programme / Generalitat de Catalunya. Part of the work was carried out at the Esther Koplowitz Center, Barcelona; Italian Ministry of Health RF-201602362288 to PG; the Comisión Honoraria de Lucha Contra el Cáncer, CSIC, Fundación Manuel Pérez, Montevideo, Uruguay; Francisco Cuellar is supported by a scholarship awarded by CONACYT, Mexico (152256/158706).

\section{Availability of data and materials}

Data will be made available upon request. This study involved human subjects and to protect the privacy of study participants, data requests will be reviewed by the GenoMEL Steering Committee. Requests for data related to this publication should be directed to Dr. Peter Kanetsky at peter. kanetsky@moffitt.org.

Ethics approval and consent to participate

Ethics approval was granted by the institutional review board (IRBs) of each recruitment center included in the study: Comité Ético de Investigación Clínica del Hospital Clinic de Barcelona (Barcelona, ES); the Danish Ethics 
Committee for the capital region of Denmark (Copenhagen, DK); Comitato Etico Azienda Ospedaliera Universitaria San Martino (Genoa, IT); North-East Yorkshire Research Ethics Committee (Leeds, UK); De Commissie Medische Ethiek van het LUMC (Leiden, NL); Republic of Slovenia, Ministry of Health, National Medical Ethics Committee (Ljubljana, SI); Regionala Etikprövningsnämnden i Lund, (Lund, SE); Hospital de Especialidades Centro Médico Nacional "La Raza" (Mexico City, MX); Comité de Ética del Hospital de Clínicas, Facultad de Medicina, UDELAR, (Montevideo, UY); Institutional Review Board of the University of Pennsylvania (Philadelphia, US); Universidade Federal de Ciências da Saúde de Porto Alegre (UFCSPA) (Porto Alegre, BR); Central Medical Ethics Committee of Latvia (Riga, LV); Comité de Ética Hospital Dr. Sótero del Río (Santiago, CL); AC Camargo Cancer Center Research Ethics Committee (São Paulo, BR); KI Research Ethical Committee North at the Karolinska Hospital (Stockholm, SE); New South Wales Population and Health Services Research Ethics Committee (Sydney, AU); and Institutional Review Board of Sheba Medical Center, Sackler Faculty of Medicine, Tel Aviv University (Tel Aviv, IL). Ethics approval for this work also was obtained by Advarra (H. Lee Moffitt Cancer Center and Research Institute). Written informed consent was obtained from all adult participants; and for centers recruiting minors, parental written informed consent was obtained. All research was performed in accordance with relevant regulations.

\section{Consent for publication}

Not applicable.

\section{Competing interests}

The authors have no conflicts of interest to declare that are relevant to the content of this article.

\section{Author details}

'Department of Cancer Epidemiology, Division of Population Science, H. Lee Moffitt Cancer Center \& Research Institute, 12902 Magnolia Dr., MRC 213, Tampa, FL 33612, USA. ${ }^{2}$ Medical Research Council - Cancer Unit, Division of Clinical Medicine, University of Cambridge, Cambridge, Cambridgeshire, UK. ${ }^{3}$ Department of Pathology, School of Medicine, Universidade de São Paulo (USP), São Paulo, SP, Brazil. ${ }^{4}$ Department of Biostatistics, Epidemiology, and Informatics, University of Pennsylvania, Philadelphia, PA, USA. ${ }^{5}$ SWOG Statistics and Data Management Center, Public Health Sciences Division, Fred Hutchinson Cancer Research Center, Seattle, WA, USA. ${ }^{6}$ Department of Dermatology, Sheba Medical Center, Tel Hashomer, Israel. ${ }^{7}$ Sackler Faculty of Medicine, Tel Aviv University, Tel Aviv, Israel. ${ }^{8}$ Servicio de Dermatología, Hospital Dr. Sótero del Río, Santiago de Chile, Chile. Institute of Medical Research at St James's, University of Leeds, Leeds, UK. ${ }^{10}$ Department of Internal Medicine and Medical Specialties, University of Genoa, Genoa, Italy. ${ }^{11}$ Departamento de Ciencias Básicas, Escuela de Medicina Universidad de Monterrey, Monterrey, Mexico. ${ }^{12}$ Melanoma Unit, Dermatology Department, Hospital Clinic Barcelona, Institut de Investigacions biomediques August Pi Sunyer (IDIBAPS), Universitat de Barcelona, Barcelona, Spain. ${ }^{13}$ Consejo Nacional de Ciencia y Tecnología (CONACYT), Ciudad de México, Mexico. ${ }^{14}$ Sydney School of Public Health, The University of Sydney, Sydney, New South Wales, Australia. ${ }^{15}$ Melanoma Institute Australia, The University of Sydney, Wollstonecraft, New South Wales, Australia. ${ }^{16}$ Department of Pathology and Laboratory Medicine, Hospital of the University of Pennsylvania, Philadelphia, PA, USA. ${ }^{17}$ Department of Clinical Genetics, University Hospital of Copenhagen, Copenhagen, Denmark. ${ }^{18}$ Genetics of Rare Cancers, IRCCS Ospedale Policlinico San Martino, Genoa, Italy. ${ }^{19}$ Pontifícia Universidade Católica do Rio Grande do Sul (PUCRS), Porto Alegre, RS, Brazil. ${ }^{20}$ Department of Dermatology, Leiden University Medical Centre, Leiden, The Netherlands. ${ }^{21}$ Department of Oncology-Pathology, Karolinska Institutet, Stockholm, Sweden. ${ }^{22}$ Institute of Oncology Ljubljana, Ljubljana, Slovenia. ${ }^{23}$ Westmead Institute for Medical Research, The University of Sydney, Westmead, New South Wales, Australia. ${ }^{24}$ Departments of Clinical Sciences and Surgery, Lund University Hospital, Lund, Sweden. ${ }^{25}$ Department of Pathology, Escola Paulista de Medicina, UNIFESP, São Paulo, Brazil.

${ }^{26}$ Department of Pathology, AC Camargo Cancer Center, São Paulo, Brazil.

${ }^{27}$ Unidad de Lesiones Pigmentadas, Cátedra de Dermatología, Hospital de Clínicas, Universidad de la República, Montevideo, Uruguay. ${ }^{28} \mathrm{John}$ Curtin School of Medical Research, Australian National University, Canberra, ACT, Australia. ${ }^{29}$ Pontificia Universidad Católica de Chile, Santiago de Chile, Chile. ${ }^{30}$ Skin Cancer Department, AC Camargo Cancer Center, São Paulo, Brazil.

${ }^{31}$ Latvian Biomedical Research and Study Centre, Riga, Latvia. ${ }^{32}$ Centro de
Investigacion Biomedica en Red de Enfermedades Raras (CIBERER), Instituto de Salud Carlos III, Barcelona, Spain. ${ }^{33}$ Departament de Medicina, Universitat de Barcelona, Barcelona, Spain. ${ }^{34}$ Dystrophic Epidermolysis Bullous Research Association Mexico, Monterrey, Mexico.

\section{Received: 22 October 2020 Accepted: 10 February 2021 Published online: 23 April 2021}

\section{References}

1. Erdmann F, Lortet-Tieulent J, Schuz J, Zeeb H, Greinert R, Breitbart EW, Bray F. International trends in the incidence of malignant melanoma 1953-2008-are recent generations at higher or lower risk? Int J Cancer. 2013;132(2):385400.

2. Loria D, Abriata MG, Santoro F, Latorre C. Cutaneous melanoma in Argentina: an analysis of its characteristics and regional differences. Ecancermedicalscience. 2020;14:1017.

3. Wainstein AJA, Duprat Neto JP, Enokihara MY, Brechtbuhl ER, Riccardi F, Landman G, de Melo AC, de Lima W, Munhoz RR, Dunshee De Abranches Oliveira Santos Filho I, et al. Demographic, clinical, and pathologic features of patients with cutaneous melanoma: final analysis of the Brazilian melanoma group database. JCO Glob Oncol. 2020;6:575-82.

4. Gandini S, Sera F, Cattaruzza MS, Pasquini P, Picconi O, Boyle P, Melchi CF. Meta-analysis of risk factors for cutaneous melanoma: II. Sun exposure. Eur J Cancer. 2005;41(1):45-60

5. Heerfordt IM, Philipsen PA, Larsen BO, Wulf HC. Long-term trend in sunscreen use among beachgoers in Denmark. Acta Derm Venereol. 2017; 97(10):1202-5

6. Koch S, Pettigrew S, Minto C, Slevin T, Strickland M, Lin C, Jalleh G. Trends in sun-protection behaviour in Australian adults 2007-2012. Australas $J$ Dermatol. 2017;58(2):111-6.

7. Rodvall $Y$, Wahlgren CF, Wiklund K. Future reduction of cutaneous malignant melanoma due to improved sun protection habits and decreased common melanocytic nevi density among Swedish children?: a follow-up from 2002 to 2012. Eur J Cancer. 2019;118:149-55.

8. Tabbakh T, Volkov A, Wakefield M, Dobbinson S. Implementation of the SunSmart program and population sun protection behaviour in Melbourne, Australia: results from cross-sectional summer surveys from 1987 to 2017. PLoS Med. 2019;16(10):e1002932.

9. Begg $C B$, Hummer A, Mujumdar U, Armstrong BK, Kricker A, Marrett LD, Millikan RC, Gruber SB, Anton-Culver H, Klotz JB, et al. Familial aggregation of melanoma risks in a large population-based sample of melanoma cases. Cancer Causes Control. 2004;15(9):957-65.

10. Diao DY, Lee TK. Sun-protective behaviors in populations at high risk for skin cancer. Psychol Res Behav Manag. 2013;7:9-18.

11. Shuk E, Burkhalter JE, Baguer CF, Holland SM, Pinkhasik A, Brady MS, Coit DG, Ariyan CE, Hay JL. Factors associated with inconsistent sun protection in first-degree relatives of melanoma survivors. Qual Health Res. 2012;22(7): 934-45.

12. Glenn BA, Lin T, Chang LC, Okada A, Wong WK, Glanz K, Bastani R. Sun protection practices and sun exposure among children with a parental history of melanoma. Cancer Epidemiol Biomarkers Prev. 2015;24(1): 169-77.

13. Wu YP, Parsons BG, Mooney R, Aspinwall LG, Cloyes K, Hay JL, Kohlmann W, Grossman D, Leachman SA. Barriers and facilitators to melanoma prevention and control behaviors among at-risk children. J Community Health. 2018; 43(5):993-1001.

14. Koch S, Pettigrew S, Hollier LP, Slevin T, Strickland M, Minto C, Jalleh G, Lin C. Trends in Australian adolescents' sun-protection behaviours: implications for health campaigns. Aust N Z J Public Health. 2016;40(5):468-73.

15. Gorig T, Diehl K, Greinert R, Breitbart EW, Schneider S. Prevalence of sunprotective behaviour and intentional sun tanning in German adolescents and adults: results of a nationwide telephone survey. J Eur Acad Dermatol Venereol. 2018;32(2):225-35.

16. Whiteman DC, Whiteman CA, Green AC. Childhood sun exposure as a risk factor for melanoma: a systematic review of epidemiologic studies. Cancer Causes Control. 2001;12(1):69-82.

17. Green AC, Wallingford SC, McBride P. Childhood exposure to ultraviolet radiation and harmful skin effects: epidemiological evidence. Prog Biophys Mol Biol. 2011;107(3):349-55.

18. Fischer AH, Wang TS, Yenokyan G, Kang S, Chien AL. Sunburn and sunprotective behaviors among adults with and without previous 
nonmelanoma skin cancer (NMSC): a population-based study. J Am Acad Dermatol. 2016;75(2):371-379 e375.

19. Savoye I, Olsen CM, Whiteman DC, Bijon A, Wald L, Dartois L, ClavelChapelon F. Boutron-Ruault MC, Kvaskoff M. Patterns of ultraviolet radiation exposure and skin cancer risk: the E3N-SunExp study. J Epidemiol. 2018; 28(1):27-33.

20. Centers for Disease C, Prevention. Sunburn and sun protective behaviors among adults aged 18-29 years--United States, 2000-2010. MMWR Morb Mortal Wkly Rep. 2012;61(18):317-22.

21. Gavin A, Boyle R, Donnelly D, Donnelly C, Gordon S, McElwee G, O'Hagan A. Trends in skin cancer knowledge, sun protection practices and behaviours in the Northern Ireland population. Eur J Public Health. 2012;22(3):408-12.

22. Ghiasvand R, Lund E, Edvardsen K, Weiderpass E, Veierod MB. Prevalence and trends of sunscreen use and sunburn among Norwegian women. $\mathrm{Br}$. Dermatol. 2015;172(2):475-83.

23. Jones SE, Saraiya M, Miyamoto J, Berkowitz Z. Trends in sunscreen use among U.S. high school students: 1999-2009. J Adolesc Health. 2012;50(3): 304-7.

24. Makin JK, Warne CD, Dobbinson SJ, Wakefield MA, Hill DJ. Population and age-group trends in weekend sun protection and sunburn over two decades of the SunSmart programme in Melbourne, Australia. Br J Dermatol. 2013;168(1):154-61.

25. Volkov A, Dobbinson S, Wakefield M, Slevin T. Seven-year trends in sun protection and sunburn among Australian adolescents and adults. Aust N Z J Public Health. 2013;37(1):63-9.

26. Azzarello LM, Dessureault S, Jacobsen PB. Sun-protective behavior among individuals with a family history of melanoma. Cancer Epidemiol Biomarkers Prev. 2006;15(1):142-5.

27. Wu YP, Parsons BG, Aspinwall LG, Hay JL, Boucher KM, Caputo H, Mooney R, Grossman D, Leachman SA. Parent and child perspectives on perceived barriers to child sun protection and their association with sun protection strategies among children of melanoma survivors. Pediatr Dermatol. 2019; 36(3):317-23.

28. Tripp MK, Peterson SK, Prokhorov AV, Shete SS, Lee JE, Gershenwald JE, Gritz ER. Correlates of sun protection and sunburn in children of melanoma survivors. Am J Prev Med. 2016;51(3):e77-85.

29. Kricker A, Armstrong BK, Goumas C, Litchfield M, Begg CB, Hummer AJ, Marrett LD, Theis B, Millikan RC, Thomas N, et al. Ambient UV, personal sun exposure and risk of multiple primary melanomas. Cancer Causes Control. 2007;18(3):295-304.

30. Kricker A, Vajdic CM, Armstrong BK. Reliability and validity of a telephone questionnaire for estimating lifetime personal sun exposure in epidemiologic studies. Cancer Epidemiol Biomarkers Prev. 2005;14(10): 2427-32.

31. Sunscreen: A History [https://www.nytimes.com/2010/06/24/fashion/24 skinside.html]. Accessed 22 June 2020

32. Siskind V, Aitken J, Green A, Martin N. Sun exposure and interaction with family history in risk of melanoma, Queensland, Australia. Int J Cancer. 2002; 97(1):90-5.

33. Elwood JM. Melanoma and sun exposure: contrasts between intermittent and chronic exposure. World J Surg. 1992;16(2):157-65.

34. Vogel Rl, Strayer LG, Engelman L, Nelson HH, Blaes AH, Anderson KE, Lazovich D. Sun exposure and protection behaviors among long-term melanoma survivors and population controls. Cancer Epidemiol Biomarkers Prev. 2017;26(4):607-13.

35. Lai YC, Yew YW. Sunburns and sun-protective behaviors after a diagnosis of melanoma. Skinmed. 2018;16(6):379-83.

36. Rodriguez-Acevedo AJ, Green AC, Sinclair C, van Deventer E, Gordon LG. Indoor tanning prevalence after the International Agency for Research on Cancer statement on carcinogenicity of artificial tanning devices: systematic review and meta-analysis. Br J Dermatol. 2020;182(4): 849-59.

37. Indoor Tanning Restrictions for Minors | A State-By-State Comparison [https://www.ncsl.org/research/health/indoor-tanning-restrictions.aspx]. Accessed 22 June 2020.

38. Bowers JM, Geller AC, Schofield E, Li Y, Hay JL. Indoor tanning trends among US adults, 2007-2018. Am J Public Health. 2020;110(6):823-8.

39. Guy GP Jr, Watson M, Seidenberg AB, Hartman AM, Holman DM, Perna F. Trends in indoor tanning and its association with sunburn among US adults. J Am Acad Dermatol. 2017;76(6):1191-3.
40. Branstrom R, Kasparian NA, Affleck P, Tibben A, Chang YM, Azizi E, BaronEpel O, Bergman W, Chan M, Davies J, et al. Perceptions of genetic research and testing among members of families with an increased risk of malignant melanoma. Eur J Cancer. 2012;48(16):3052-62.

\section{Publisher's Note}

Springer Nature remains neutral with regard to jurisdictional claims in published maps and institutional affiliations.

\section{Ready to submit your research? Choose BMC and benefit from:}

- fast, convenient online submission

- thorough peer review by experienced researchers in your field

- rapid publication on acceptance

- support for research data, including large and complex data types

- gold Open Access which fosters wider collaboration and increased citations

- maximum visibility for your research: over $100 \mathrm{M}$ website views per year

At $\mathrm{BMC}$, research is always in progress.

Learn more biomedcentral.com/submissions 